\title{
A Deep-learned Type-3 Fuzzy System and Its Application in Modeling Problems
}

\author{
Man-Wen Tian', Ardashir Mohammadzadeh², Jafar Tavoosi ${ }^{3}$, \\ Saleh Mobayen ${ }^{4}$, Jihad H. Asad ${ }^{5}$, Oscar Castillo ${ }^{6}$, Annámaria R. \\ Várkonyi-Kóczy ${ }^{7}$
}

${ }^{1}$ National key project laboratory, Jiangxi University of Engineering, Xinyu 338000, China; E-mail: mwtian@ygu.edu.cn

${ }^{2}$ Department of Electrical Engineering, Faculty of Engineering University of Bonab, Bonab, Iran; Email: a.mzadeh@ubonab.ac.ir

${ }^{3}$ Department of Electrical Engineering, Faculty of Engineering, Ilam University, Ilam, Iran; Email: jtavoosi@aut.ac.ir

${ }^{4}$ Future Technology Research Center, National Yunlin University of Science and Technology, Douliu 64002, Taiwan; Email: mobayens@yuntech.edu.tw

${ }^{5}$ Department of Physics, Faculty of Applied Sciences, Palestine Technical University, Tulkarm, Palestine; Email: j.asad@ptuk.edu.ps

${ }^{6}$ Division of Graduate Studies and Research, Tijuana Institute of Technology, Tijuana, Mexico. Email: ocastillo@tectijuana.mx

${ }^{7}$ Institute of Software Design and Software Development, Óbuda University, Budapest, Hungary; Email: varkonyi-koczy@uni-obuda.hu Department of Informatics, J. Selye University, Komarno, Slovakia

Abstract: The modeling problem is one of the important topics in engineering applications. In various applications, it is required to find a mathematical model to represent the relationship between output and the associated input variables. In this study, an approach on basis of a new deep learned type-3 (T3) fuzzy logic system (FLS) is introduced. The modeling of $\mathrm{CO}_{2}$ solubility on basis of temperature, molality of $\mathrm{NaCl}$, and pressure is considered as an application. The monitoring of carbon dioxide $\left(\mathrm{CO}_{2}\right)$ solubility in brine is one of the effective approaches in carbon capture and sequestration technique to reduce it in the atmosphere. A new hybrid learning method is presented to optimize the suggested model. The new adaptation laws are carry-out to tune the rule parameters and centers of membership functions (MFs). The values of horizontal slices and $\alpha$-cuts are learned by the unscented Kalman filter $(U K F)$. By the real-world experimental data sets, several statistical examinations, and comparison with conventional well-known fuzzy neural networks (NNs) and learning methods, the reliability and good performance of the suggested method are demonstrated. Also, the sensitivity of the input variables is analyzed by the use of the Sobol approach. 
Keywords: Carbon dioxide solubility; Fuzzy logic systems; Learning algorithm; Estimation performance; Kalman filter

\section{Introduction}

Modeling problem is one of the important topics in engineering applications. In various applications, it is required to find a mathematical model to represent the relationship between output and associated input variables. In recent years, one of the main approaches that have been frequently reported in the literature to decrease carbon dioxide $\left(\mathrm{CO}_{2}\right)$ is carbon capture and sequestration (CCS). The solubility of $\mathrm{CO}_{2}$ in brine is the basic factor in the CCS. Then forecasting the solubility of $\mathrm{CO}_{2}$ with the desired accuracy is an important research topic [1].

For modeling and forecasting the solubility of $\mathrm{CO}_{2}$, many approaches have been presented. For example, in [2], some experimental data in a special pressure and temperature is obtained, and then using Peng-Robinson equations a mathematical model is extracted. In [3], a thermodynamic model by the use of Soave-RedlichKwong equations is presented and its accuracy is discussed by some experimental data. The reliability of various existing models is discussed in [4], and the effect of pressure is studied. In [5], the development of scientific models for $\mathrm{CO}_{2}$ is reviewed and the practical conditions to evaluate the capability of various models are discussed. In [6], by the use of VPT, CPA-SRK72, and PC-SAFT equations a model is presented to predict the solubility of $\mathrm{CO}_{2}$ in brine and water, and the sensitivity of $\mathrm{CO}_{2}$ solubility with respect to the temperature and pressure is investigated. In [7], some experimental data at high pressure for $\mathrm{CO}_{2}$ solubility in brine is measured and then by Whitson equations, a model is developed. In [8], using Setschenow coefficients a developed model is presented and its performance is compared with other models and also the behavior of $\mathrm{CO}_{2}$ solubility in versus of temperature is investigated. In [9], some new experimental data is presented and the Patel-Teja equation is taken to account to construct a model and its agreement with gathered data is investigated. In [10], by the staticanalytic method some new experimental data is extracted and two models are developed by the use of Robinson Cubic and Soreide and Whitson equations. In [11], the Soave-RedlichKwong equation is developed to obtain a model, and its superiority against the Peng-Robinson model is studied.

The machine learning techniques and intelligent systems such as FLS and neural networks have been successfully employed on various engineering problems such as complex problem applications [12], risk solving [13], prediction problems [14], optimization [15], susceptibility analysis [16], classification problems [17], control systems [18], among many others. However, rarely studies can be found in literature about modeling and forecasting $\mathrm{CO}_{2}$ solubility by these techniques. For example, in [19], the least-square support-vector machine (LSSVM) is 
proposed to estimate the $\mathrm{CO}_{2}$ solubility, and its effectiveness is investigated in versus of optimized FLS by particle swarm optimization (PSO) technique. Similar to [19], the superiority of LSSVM against multi-layer perceptron (MLP) and radial basis function NN (RBF) is shown in [20]. It is declared that the R-squared value for LSSVM is 0.991 is versus of 0.964 and 0.916 for MLP and RBF, respectively. In [21], the Adaptive Boosting (AdaBoost) algorithm and NNs concept are combined to establish a model and its carnality is compared with LSSVMs. Similarly, in [22], the superiority of the AdaBoost technique is investigated by comparison with MLPs and LSSVMs. In [23], an Extra Trees model is developed and it is declared that the performance of the suggested method is better than FLS and NN approaches. In [24], two systems on basis of RBF and FLS are optimized by the genetic algorithm (GA) to estimate the $\mathrm{CO}_{2}$ solubility. In [25], an FLS is optimized to approximate the $\mathrm{CO}_{2}$ solubility and its accuracy and convergence velocity are studied. In [26], the FLS, NN, and selforganizing map techniques are combined to construct an intelligent model to predict $\mathrm{CO}_{2}$ as a function of economic enhancement and energy consumption. In [27], an FLS model is developed, and by the use of Monte-Carlo method the sensitivity of the model with respect to pressure and temperature is investigated. In [28], an FLS is tuned by PSO algorithm and it is applied for $\mathrm{CO}_{2}$ and methane solubility. In [29], the $\mathrm{CO}_{2}$ viscosity is modeled by MLP and the parameters of MLP are optimized by the Levenberg-Marquardt (LM) algorithm and it is shown that the use of LM results in better accuracy in contrast to conjugate gradient algorithm. Similarly, in [30], an FLS is learned by various evolutionary optimization methods such as artificial bee colony (ABC), PSO, and GA, and the superiority of optimized FLS by PSO is shown. In [31], the effectiveness of the LSSVM in estimating the $\mathrm{CO}_{2}$ solubility is studied and the influence of salinity, pressure, and temperature is analyzed. In [32], a model is developed by the use of GA and support vector machine (SVM), and its proficiency is examined in versus of NN-based approaches. In [33-36], decision making techniques are used for modeling problems in engineering applications. Recently it has been shown that the high-order FLSs are more effective than conventional NN-based approaches in modeling complicated nonlinear systems. However, it has not been used in CCS problem.

Motivated by the above literature review, in this paper, a new approach on basis of deep learned type-3 FLS is proposed to construct a model for $\mathrm{CO}_{2}$ solubility estimation. For the first time, in addition to the rule parameters, the centers of MFs and level of horizontal slices are also learned. The effectiveness of the suggested method is examined by several statistical analyses and comparisons with conventional well-known methods. The main highlights are:

- For the first time, a deep learned T3-FLS is proposed.

- A hybrid learning method is presented such that, in addition to the rule parameters, the centers of MFs and level of horizontal slices are also learned.

- A new approach is presented to investigate the sensitivity of input variables. 
- Several statistical analyses are provided to verify the effectiveness of the suggested T3-FLS.

- Some comparisons with various fuzzy neural networks and learning algorithms are provided to show the superiority of the suggested T3-FLS and hybrid learning method.

\section{Proposed Type-3 FLS}

\subsection{General View}

The type-3 FLS [37], is the generalization of the type-2 FLS that has more capacity to cope with uncertainties. A general view on the suggested T3-FLS is depicted in Figure 1. In T3-FLSs, as shown in Figure 2, the secondary membership function (MF) is also a type-2 MF. Then the upper and lower bounds of memberships are not constant in contrast to the type-2 MFs. This features cause that more level of uncertainties can be handled by type-3 MFs.

\subsection{Structure}

In this section, the process of computing is explained:

1) The inputs are $T, P$, and $M$, which represents temperature $\left({ }^{\circ} K\right)$, pressure (bar), and molality of $\mathrm{NaCl}\left(\mathrm{mol} \cdot \mathrm{kg}^{-1}\right)$, respectively.

2) For each inputs $T, P$, and $M$, two membership functions (MFs) are considered. The MFs of inputs $T, P$ and $M$ are denoted as $\tilde{S}_{T}^{1}-\tilde{S}_{T}^{2}$ and $S_{P}^{1}-\tilde{S}_{P}^{2}$, respectively. Each MF is horizontally sliced into $n$ levels as shown in Figure 2. As shown in Figure 3, for each input, the upper and lower memberships for horizontal slice level $\alpha_{h}$, are computed. For input $T$ the upper and lower memberships at horizontal splice level $\alpha_{h}$ are obtained as:

$\bar{\mu}_{\tilde{S}_{T}^{j} \mid \bar{\alpha}_{h}}(T)=\exp \left(-\frac{\left(T-c_{\tilde{S}_{T}^{j} \mid \bar{\alpha}_{h}}\right)^{2}}{\bar{\sigma}_{\tilde{S}_{T}^{j} \mid \bar{\alpha}_{h}}^{2}}\right), \bar{\mu}_{\tilde{S}_{T}^{j} \mid \underline{\alpha}_{h}}(T)=\exp \left(-\frac{\left(T-c_{\tilde{S}_{T}^{j} \mid \underline{\alpha}_{h}}\right)^{2}}{\bar{\sigma}_{\tilde{S}_{T}^{j} \mid \underline{\alpha}_{h}}^{2}}\right)$ 


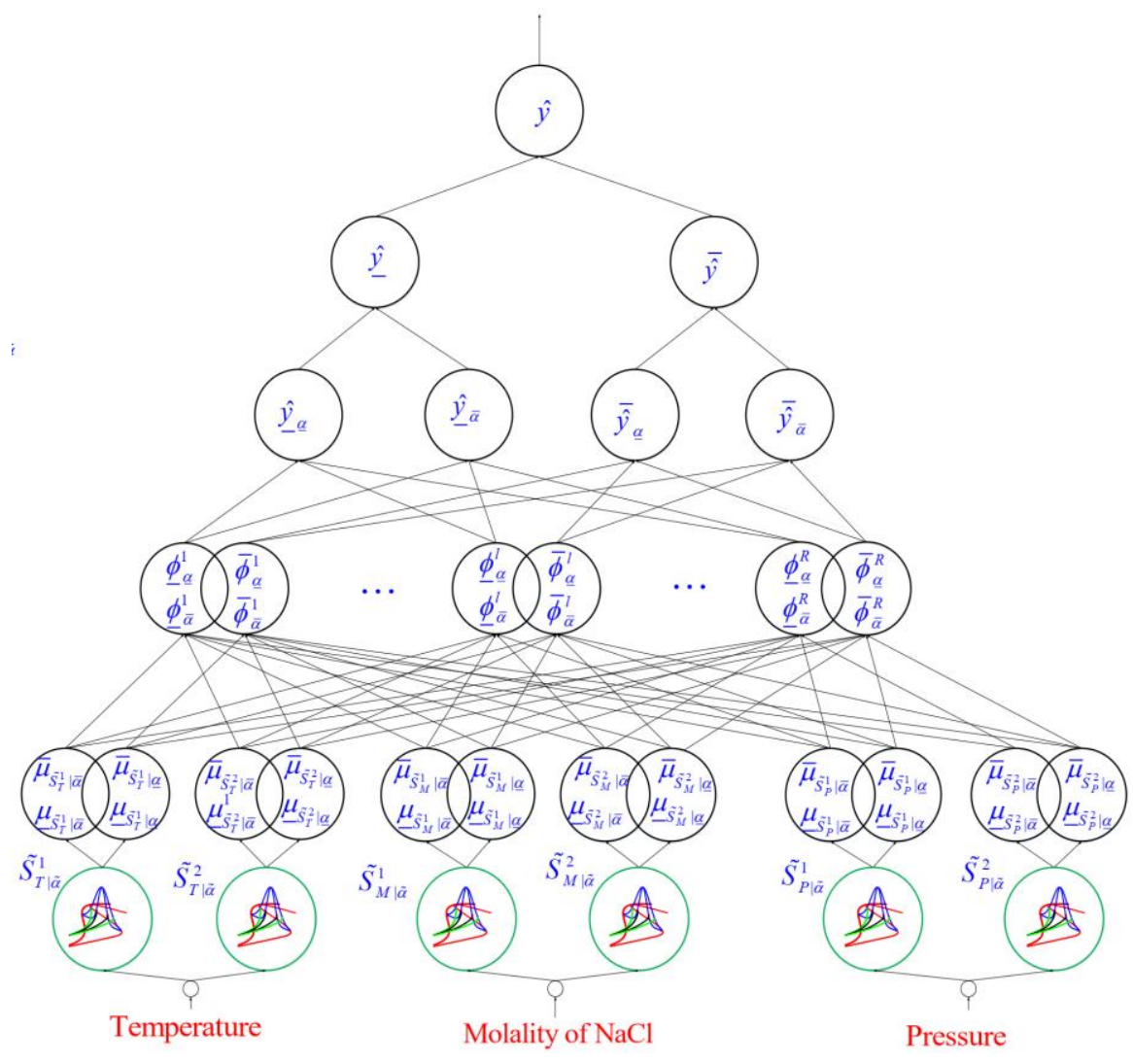

Figure 1

A general view on the suggested type-3 fuzzy logic system

$\underline{\mu}_{\tilde{S}_{T}^{j} \mid \bar{\alpha}_{h}}(T)=\exp \left(-\frac{\left(T-c_{\tilde{S}_{T}^{j} \mid \bar{\alpha}_{h}}\right)^{2}}{\underline{\sigma}_{\tilde{S}_{T}^{j} \mid \bar{\alpha}_{h}}^{2}}\right), \underline{\mu}_{\tilde{S}_{T}^{j} \mid \underline{\alpha}_{h}}(T)=\exp \left(-\frac{\left(T-c_{\tilde{S}_{T}^{j} \mid \underline{\alpha}_{h}}\right)^{2}}{\underline{\sigma}_{\tilde{S}_{T}^{j} \mid \underline{\alpha}_{h}}^{2}}\right)$

where, $h=1, \ldots, n, j=1,2, c_{\tilde{S}_{T}^{j} \mid \bar{\alpha}_{h}}$ is the center of MF $\tilde{S}_{T}^{j} \mid \bar{\alpha}_{h}, \bar{\sigma}_{\tilde{S}_{T}^{j} \mid \bar{\alpha}_{h}}$, and ${\underline{S_{\tilde{T}}^{j}} \mid \bar{\alpha}_{h}}$ are the upper and lower standard divisions for $\tilde{S}_{T}^{j} \mid \bar{\alpha}_{h}$. For input $P$, one has:

$\bar{\mu}_{\tilde{S}_{P}^{j} \mid \bar{\alpha}_{h}}(P)=\exp \left(-\frac{\left(P-c_{\tilde{S}_{p}^{j} \mid \bar{\alpha}_{h}}\right)^{2}}{\bar{\sigma}_{\tilde{S}_{P}^{j} \mid \bar{\alpha}_{h}}^{2}}\right), \bar{\mu}_{\tilde{S}_{P}^{j} \mid \underline{\alpha}_{h}}(P)=\exp \left(-\frac{\left(P-c_{\tilde{S}_{P}^{j} \mid \underline{\alpha}_{h}}\right)^{2}}{\bar{\sigma}_{\tilde{S}_{P}^{j} \mid \underline{\alpha}_{h}}^{2}}\right)$ (3) 


$$
\underline{\mu}_{\tilde{S}_{P}^{j} \mid \bar{\alpha}_{h}}(P)=\exp \left(-\frac{\left(P-c_{\tilde{S}_{P}^{j} \mid \bar{\alpha}_{h}}\right)^{2}}{\underline{\sigma}_{\tilde{S}_{P}^{j} \mid \bar{\alpha}_{h}}^{2}}\right), \underline{\mu}_{\tilde{S}_{P}^{j} \mid \underline{\alpha}_{h}}(P)=\exp \left(-\frac{\left(P-c_{\tilde{S}_{P}^{j} \mid \underline{\alpha}_{h}}\right)^{2}}{\underline{\sigma}_{\tilde{S}_{P}^{j} \mid \underline{\alpha}_{h}}^{2}}\right)
$$

where, $h=1, \ldots, n, j=1,2, c_{\tilde{S}_{P}^{j} \mid \bar{\alpha}_{h}}$ is the center of MF $\tilde{S}_{P}^{j} \mid \bar{\alpha}_{h}, \underline{\sigma}_{\tilde{S}_{P}^{j} \mid \bar{\alpha}_{h}}$ and $\underline{\sigma}_{\tilde{S}_{P}^{j} \mid \bar{\alpha}_{h}}$ are the upper and lower standard division for $\tilde{S}_{P}^{j} \mid \bar{\alpha}_{h}$. Similarly, for input $M$, one has:

$$
\begin{aligned}
& \bar{\mu}_{\tilde{S}_{M}^{j} \mid \bar{\alpha}_{h}}=\exp \left(\frac{-\left(M-c_{\tilde{S}_{M}^{j} \mid \bar{\alpha}_{h}}\right)^{2}}{\bar{\sigma}_{\tilde{S}_{M}^{j} \mid \bar{\alpha}_{h}}^{2}}\right), \bar{\mu}_{\tilde{S}_{M}^{j} \mid \underline{\alpha}_{h}}=\exp \left(\frac{-\left(M-c_{\tilde{S}_{M}^{j} \mid \underline{\alpha}_{h}}\right)^{2}}{\bar{\sigma}_{\tilde{S}_{M}^{j} \mid \underline{\alpha}_{h}}^{2}}\right) \\
& \underline{\mu}_{\tilde{S}_{M}^{j} \mid \bar{\alpha}_{h}}=\exp \left(-\frac{\left(M-c_{\tilde{S}_{M}^{j} \mid \bar{\alpha}_{h}}\right)^{2}}{\underline{\sigma}_{\tilde{S}_{M}^{j} \mid \bar{\alpha}_{h}}^{2}}\right), \underline{\mu}_{\tilde{S}_{M}^{j} \mid \underline{\alpha}_{h}}=\exp \left(-\frac{\left(M-c_{\tilde{S}_{M}^{j} \mid \underline{\alpha}_{h}}\right)^{2}}{\underline{\sigma}_{\tilde{S}_{M}^{j} \mid \underline{\alpha}_{h}}^{2}}\right)
\end{aligned}
$$

where, $h=1, \ldots, n, j=1,2, c_{\tilde{S}_{P}^{j} \mid \bar{\alpha}_{h}}$ is the center of MF $\tilde{S}_{M}^{j} \mid \bar{\alpha}_{h}, \bar{\sigma}_{\tilde{S}_{M}^{j} \mid \bar{\alpha}_{h}}$ and $\underline{\sigma}_{\tilde{S}_{M}^{j} \mid \bar{\alpha}_{h}}$ are the upper and lower standard division for $\tilde{S}_{M}^{j} \mid \bar{\alpha}_{h}$.

3) The upper rule firing at $\bar{\alpha}_{h}$ are computed as:

$$
\begin{aligned}
& \bar{\varphi}_{\bar{\alpha}_{h}}^{1}=\bar{\mu}_{\tilde{S}_{T}^{1} \mid \bar{\alpha}_{h}} \bar{\mu}_{\tilde{S}_{P}^{1} \mid \bar{\alpha}_{h}} \bar{\mu}_{\tilde{S}_{M}^{1} \mid \bar{\alpha}_{h}}, \bar{\varphi}_{\bar{\alpha}_{h}}^{2}=\bar{\mu}_{\tilde{S}_{T}^{1} \mid \bar{\alpha}_{h}} \bar{\mu}_{\tilde{S}_{P}^{1} \mid \bar{\alpha}_{h}} \bar{\mu}_{\tilde{S}_{M}^{2} \mid \bar{\alpha}_{h}} \\
& \bar{\varphi}_{\bar{\alpha}_{h}}^{3}=\bar{\mu}_{\tilde{S}_{T}^{1} \mid \bar{\alpha}_{h}} \bar{\mu}_{\tilde{S}_{P}^{2} \mid \bar{\alpha}_{h}} \bar{\mu}_{\tilde{S}_{M}^{1} \mid \bar{\alpha}_{h}}, \bar{\varphi}_{\bar{\alpha}_{h}}^{4}=\bar{\mu}_{\tilde{S}_{T}^{1} \mid \bar{\alpha}_{h}} \bar{\mu}_{\tilde{S}_{P}^{2} \mid \bar{\alpha}_{h}} \bar{\mu}_{\tilde{S}_{M}^{2} \mid \bar{\alpha}_{h}} \\
& \bar{\varphi}_{\bar{\alpha}_{h}}^{5}=\bar{\mu}_{\tilde{S}_{T}^{2} \mid \bar{\alpha}_{h}} \bar{\mu}_{\tilde{S}_{P}^{1} \mid \bar{\alpha}_{h}} \bar{\mu}_{\tilde{S}_{M}^{1} \mid \bar{\alpha}_{h}}, \bar{\varphi}_{\bar{\alpha}_{h}}^{6}=\bar{\mu}_{\tilde{S}_{T}^{2} \mid \bar{\alpha}_{h}} \bar{\mu}_{\tilde{S}_{P}^{1} \mid \bar{\alpha}_{h}} \bar{\mu}_{\tilde{S}_{M}^{2} \mid \bar{\alpha}_{h}}^{7}=\bar{\mu}_{\tilde{S}_{T}^{2} \mid \bar{\alpha}_{h}} \bar{\mu}_{\tilde{S}_{P}^{2} \mid \bar{\alpha}_{h}} \bar{\mu}_{\tilde{S}_{M}^{1} \mid \bar{\alpha}_{h}}, \bar{\varphi}_{\bar{\alpha}_{h}}^{8}=\bar{\mu}_{\tilde{S}_{T}^{2} \mid \bar{\alpha}_{h}} \bar{\mu}_{\tilde{S}_{P}^{2} \mid \bar{\alpha}_{h}} \bar{\mu}_{\tilde{S}_{M}^{2} \mid \bar{\alpha}_{h}}
\end{aligned}
$$

For upper rule firing at $\underline{\alpha}_{h}$, one has:

$$
\begin{aligned}
& \bar{\varphi}_{\underline{\alpha}_{h}}^{1}=\bar{\mu}_{\tilde{S}_{T}^{1} \mid \underline{\alpha}_{h}} \bar{\mu}_{\tilde{S}_{P}^{1} \mid \underline{\alpha}_{h}} \bar{\mu}_{\tilde{S}_{M}^{1} \mid \underline{\alpha}_{h}}, \bar{\varphi}_{\underline{\alpha}_{h}}^{2}=\bar{\mu}_{\tilde{S}_{T}^{1} \mid \underline{\alpha}_{h}} \bar{\mu}_{\tilde{S}_{P}^{1} \mid \underline{\alpha}_{h}} \bar{\mu}_{\tilde{S}_{M}^{2} \mid \underline{\alpha}_{h}} \\
& \bar{\varphi}_{\underline{\alpha}_{h}}^{3}=\bar{\mu}_{\tilde{S}_{T}^{1} \mid \underline{\alpha}_{h}} \bar{\mu}_{\tilde{S}_{P}^{2} \mid \underline{\alpha}_{h}} \bar{\mu}_{\tilde{S}_{M}^{1} \mid \underline{\alpha}_{h}}, \bar{\varphi}_{\underline{\alpha}_{h}}^{4}=\bar{\mu}_{\tilde{S}_{T}^{1} \mid \underline{\alpha}_{h}} \bar{\mu}_{\tilde{S}_{P}^{2} \mid \underline{\alpha}_{h}} \bar{\mu}_{\tilde{S}_{M}^{2} \mid \underline{\alpha}_{h}}=\bar{\mu}_{\tilde{S}_{T}^{2} \mid \underline{\alpha}_{h}} \bar{\mu}_{\tilde{S}_{P}^{1} \mid \underline{\alpha}_{h}} \bar{\mu}_{\tilde{S}_{M}^{1} \mid \underline{\alpha}_{h}}, \bar{\varphi}_{\underline{\alpha}_{h}}^{6}=\bar{\mu}_{\tilde{S}_{T}^{2} \mid \underline{\alpha}_{h}} \bar{\mu}_{\tilde{S}_{P}^{1} \mid \underline{\alpha}_{h}} \bar{\mu}_{\tilde{S}_{M}^{2} \mid \underline{\alpha}_{h}} \\
& \bar{\varphi}_{\underline{\alpha}_{h}}^{7}=\bar{\mu}_{\tilde{S}_{T}^{2} \mid \underline{\alpha}_{h}} \bar{\mu}_{\tilde{S}_{P}^{2} \mid \underline{\alpha}_{h}} \bar{\mu}_{\tilde{S}_{M}^{1} \mid \underline{\alpha}_{h}}, \bar{\varphi}_{\underline{\alpha}_{h}}^{8}=\bar{\mu}_{\tilde{S}_{T}^{2} \mid \underline{\alpha}_{h}} \bar{\mu}_{\tilde{S}_{P}^{2} \mid \underline{\alpha}_{h}} \bar{\mu}_{\tilde{S}_{M}^{2} \mid \underline{\alpha}_{h}}
\end{aligned}
$$


Similarly, the lower firing rules at upper and lower slices are computed as:

$$
\begin{aligned}
& \underline{\varphi}_{\bar{\alpha}_{h}}^{1}=\bar{\mu}_{\tilde{S}_{T}^{1} \mid \bar{\alpha}_{h}} \bar{\mu}_{\tilde{S}_{P}^{1} \mid \bar{\alpha}_{h}} \bar{\mu}_{\tilde{S}_{M}^{1} \mid \bar{\alpha}_{h}}, \underline{\varphi}_{\bar{\alpha}_{h}}^{2}=\bar{\mu}_{\tilde{S}_{T}^{1} \mid \bar{\alpha}_{h}} \bar{\mu}_{\tilde{S}_{P}^{1} \mid \bar{\alpha}_{h}} \bar{\mu}_{\tilde{S}_{M}^{2} \mid \bar{\alpha}_{h}} \\
& \underline{\varphi}_{\bar{\alpha}_{h}}^{3}=\bar{\mu}_{\tilde{S}_{T}^{1} \mid \bar{\alpha}_{h}} \bar{\mu}_{\tilde{S}_{P}^{2} \mid \bar{\alpha}_{h}} \bar{\mu}_{\tilde{S}_{M}^{1} \mid \bar{\alpha}_{h}}, \underline{\varphi}_{\bar{\alpha}_{h}}^{4}=\bar{\mu}_{\tilde{S}_{T}^{1} \mid \bar{\alpha}_{h}} \bar{\mu}_{\tilde{S}_{P}^{2} \mid \bar{\alpha}_{h}} \bar{\mu}_{\tilde{S}_{M}^{2} \mid \bar{\alpha}_{h}} \\
& \underline{\varphi}_{\bar{\alpha}_{h}}^{5}=\bar{\mu}_{\tilde{S}_{T}^{2} \mid \bar{\alpha}_{h}} \bar{\mu}_{\tilde{S}_{P}^{1} \mid \bar{\alpha}_{h}} \bar{\mu}_{\tilde{S}_{M}^{1} \mid \bar{\alpha}_{h}}, \underline{\varphi}_{\bar{\alpha}_{h}}^{6}=\bar{\mu}_{\tilde{S}_{T}^{2} \mid \bar{\alpha}_{h}} \bar{\mu}_{\tilde{S}_{P}^{1} \mid \bar{\alpha}_{h}} \bar{\mu}_{\tilde{S}_{M}^{2} \mid \bar{\alpha}_{h}}=\bar{\mu}_{\tilde{S}_{T}^{2} \mid \bar{\alpha}_{h}} \bar{\mu}_{\tilde{S}_{P}^{2} \mid \bar{\alpha}_{h}} \bar{\mu}_{\tilde{S}_{M}^{1} \mid \bar{\alpha}_{h}}, \underline{\varphi}_{\bar{\alpha}_{h}}^{8}=\bar{\mu}_{\tilde{S}_{T}^{2} \mid \bar{\alpha}_{h}} \bar{\mu}_{\tilde{S}_{P}^{2} \mid \bar{\alpha}_{h}} \bar{\mu}_{\tilde{S}_{M}^{2} \mid \bar{\alpha}_{h}} \\
& \underline{\varphi}_{\underline{\alpha}_{h}}^{3}=\bar{\mu}_{\tilde{S}_{T}^{1}\left|\underline{\alpha}_{h}\right| \underline{\alpha}_{h}} \bar{\mu}_{\tilde{S}_{P}^{1} \mid \underline{\alpha}_{h}} \bar{\mu}_{\tilde{S}_{M}^{1}\left|\underline{\alpha}_{h}^{2}\right| \underline{\alpha}_{h}}, \underline{\varphi}_{\tilde{\alpha}_{h}}^{2}=\bar{\mu}_{\tilde{S}_{T}^{1} \mid \underline{\alpha}_{h}}, \bar{\mu}_{\tilde{S}_{P}^{1} \mid \underline{\alpha}_{h}}, \underline{\varphi}_{\tilde{S}_{\tilde{S}_{h}}^{2} \mid \underline{\alpha}_{h}}^{4}=\bar{\mu}_{\tilde{S}_{T}^{1} \mid \underline{\alpha}_{h}} \bar{\mu}_{\tilde{S}_{P}^{2} \mid \underline{\alpha}_{h}} \bar{\mu}_{\tilde{S}_{T}^{2} \mid \underline{\alpha}_{h}} \bar{\mu}_{\tilde{S}_{P}^{1} \mid \underline{\alpha}_{h}} \bar{\mu}_{\tilde{S}_{M}^{1} \mid \underline{\alpha}_{h}}, \underline{\varphi}_{\underline{\alpha}_{h}}^{6}=\bar{\mu}_{\tilde{S}_{T}^{2} \mid \underline{\alpha}_{h}} \bar{\mu}_{\tilde{S}_{P}^{1} \mid \underline{\alpha}_{h}} \bar{\mu}_{\tilde{S}_{M}^{2} \mid \underline{\alpha}_{h}} \\
& \underline{\varphi}_{\underline{\alpha}_{h}}^{7}=\bar{\mu}_{\tilde{S}_{T}^{2} \mid \underline{\alpha}_{h}} \bar{\mu}_{\tilde{S}_{P}^{2} \mid \underline{\alpha}_{h}} \bar{\mu}_{\tilde{S}_{M}^{1} \mid \underline{\alpha}_{h}}, \underline{\varphi}_{\alpha_{h}}^{8}=\bar{\mu}_{\tilde{S}_{T}^{2} \mid \underline{\alpha}_{h}} \bar{\mu}_{\tilde{S}_{P}^{2} \mid \underline{\alpha}_{h}} \bar{\mu}_{\tilde{S}_{M}^{2} \mid \underline{\alpha}_{h}}
\end{aligned}
$$

4) For the first type-reduction, the upper and lower of estimated output are computed as:

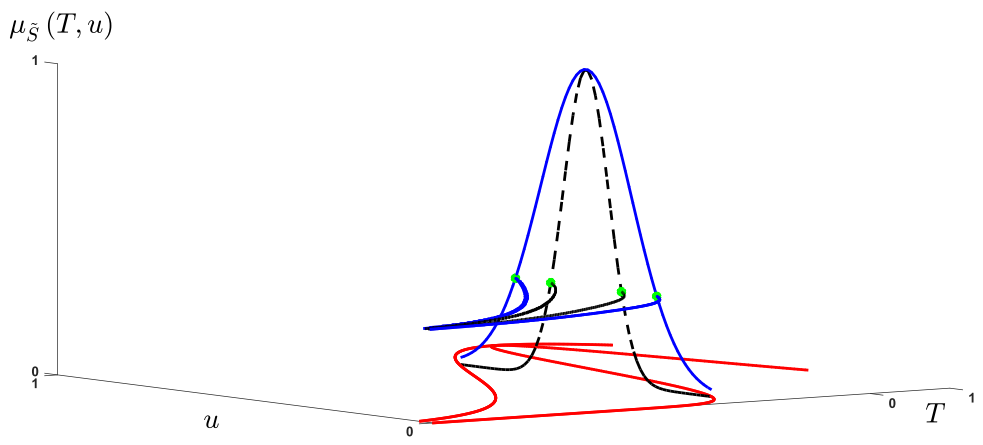

Figure 2

The horizontal slices of type-3 MF 


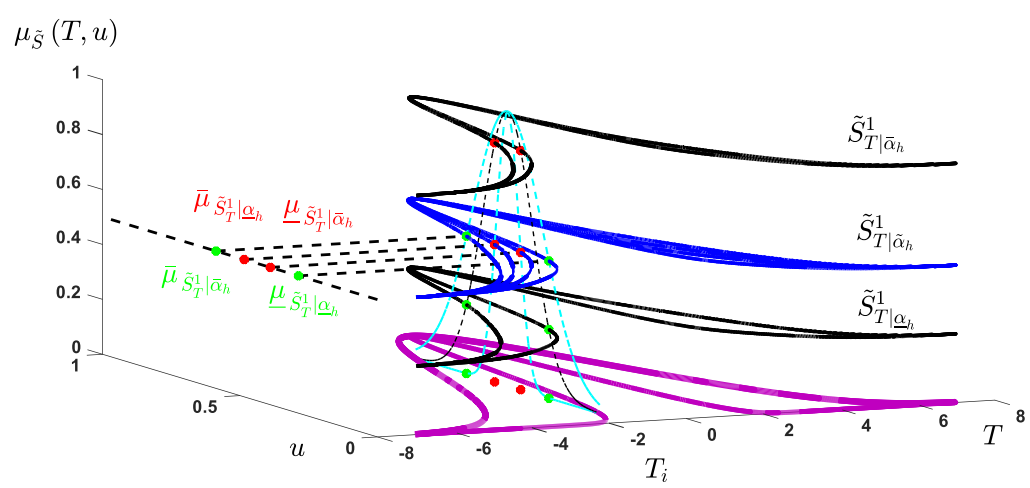

Figure 3

Representation of upper and lower memberships by two horizontal slices

$\overline{\hat{y}}_{\bar{\alpha}_{h}}=\frac{\sum_{l=1}^{R} \bar{\varphi}_{\bar{\alpha}_{h}}^{l} \bar{\theta}_{l}}{\sum_{l=1}^{R}\left(\bar{\varphi}_{\bar{\alpha}_{h}}^{l}+\underline{\varphi}_{\bar{\alpha}_{h}}^{l}\right)}, \overline{\hat{y}}_{\underline{\alpha}_{h}}=\frac{\sum_{l=1}^{R} \bar{\varphi}_{\underline{\alpha}_{h}}^{l} \bar{\theta}_{l}}{\sum_{l=1}^{R}\left(\bar{\varphi}_{\underline{\alpha}_{h}}^{l}+\underline{\varphi}_{\alpha_{h}}^{l}\right)}$

$\underline{\hat{y}}_{\bar{\alpha}_{h}}=\frac{\sum_{l=1}^{R} \underline{\varphi}_{\bar{\alpha}_{h}}^{l} \underline{\theta}_{l}}{\sum_{l=1}^{R}\left(\bar{\varphi}_{\bar{\alpha}_{h}}^{l}+\underline{\varphi}_{\bar{\alpha}_{h}}^{l}\right)}, \underline{\hat{y}}_{\underline{\alpha}_{h}}=\frac{\sum_{l=1}^{R} \bar{\varphi}_{\underline{\alpha}_{h}}^{l} \underline{\theta}_{l}}{\sum_{l=1}^{R}\left(\underline{\varphi}_{\underline{\alpha}_{h}}^{l}+\underline{\varphi}_{\underline{\alpha}_{h}}^{l}\right)}$

where, $R=8$ is the number of rules, $\underline{\theta}_{l}$ and $\bar{\theta}_{l}$ are the lower and upper of $l-$ th rule parameters.

5) For the second type-reduction, one has:

$$
\overline{\hat{y}}=\frac{\sum_{h=1}^{n} \bar{\alpha}_{h} \overline{\hat{y}}_{\bar{\alpha}_{h}}}{\sum_{h=1}^{n}\left(\bar{\alpha}_{h}+\underline{\alpha}_{h}\right)}+\frac{\sum_{h=1}^{n} \underline{\alpha}_{h} \overline{\hat{y}}_{\underline{\alpha}_{h}}}{\sum_{h=1}^{n}\left(\bar{\alpha}_{h}+\underline{\alpha}_{h}\right)}, \underline{\hat{y}}=\frac{\sum_{h=1}^{n} \bar{\alpha}_{h} \underline{\hat{y}}_{\bar{\alpha}_{h}}}{\sum_{h=1}^{n}\left(\bar{\alpha}_{h}+\underline{\alpha}_{h}\right)}+\frac{\sum_{h=1}^{n} \underline{\alpha}_{h} \underline{\hat{y}}_{\underline{\alpha}_{h}}}{\sum_{h=1}^{n}\left(\bar{\alpha}_{h}+\underline{\alpha}_{h}\right)}
$$

6) The output $\hat{y}$ is the estimated solubility $\left(\mathrm{mol} \cdot \mathrm{kg}^{-1}\right)$ that is computed as:

$$
\hat{y}=\frac{\overline{\hat{y}}+\underline{\hat{y}}}{2}
$$




\section{Learning Algorithm}

In this section, the rule parameters, the centers of MFs, and the values of horizontal slices are tuned.

\subsection{Tuning of Rule Parameters}

The rule parameters are tuned by the EKF algorithm such that the following cost function are to be minimized:

$J=\frac{1}{2}\left(y_{d}-\hat{y}\right)^{2}$

where, $y_{d}$ is the desired solubility $\left(\mathrm{mol} \cdot \mathrm{kg}^{-1}\right)$ and $\hat{y}$ is the output of the suggested T3-FLS that represents the estimated solubility $\left(\mathrm{mol} \cdot \mathrm{kg}^{-1}\right)$. The tuning laws for the upper and lower rule parameters $\bar{\theta}$ and $\underline{\theta}$ are given as:

$\bar{\theta}(t)=\bar{\theta}(t-1)+\bar{\pi}(t) \bar{\psi}(t)\left(y_{d}-\hat{y}\right), \underline{\theta}(t)=\underline{\theta}(t-1)+\underline{\pi}(t) \underline{\psi}(t)\left(y_{d}-\hat{y}\right)$

where, $\bar{\pi}$ and $\underline{\pi}(t)$ are the corresponding covariance matrices for $\bar{\theta}$ an $\underline{\theta}$, respectively. The terms $\bar{\psi}(t)$ and $\underline{\psi}(t)$ are defined as:

$\bar{\psi}=\left[\bar{\psi}_{1}, \ldots, \bar{\psi}_{l}, \ldots, \bar{\psi}_{R}\right]^{T}, \underline{\psi}=\left[\underline{\psi}_{1}, \ldots, \underline{\psi}_{l}, \ldots, \underline{\psi}_{R}\right]^{T}$

where, $\bar{\psi}(t)$ and $\underline{\psi}(t)$ are:

$$
\begin{aligned}
& \bar{\psi}_{l}=\frac{\partial \hat{y}}{\partial \bar{\theta}_{l}}=\frac{\partial \hat{y}}{\partial \overline{\hat{y}}} \frac{\partial \overline{\hat{y}}}{\partial \bar{\theta}_{l}}=\frac{\partial \hat{y}}{\partial \overline{\hat{y}}} \frac{\partial \overline{\hat{y}}^{\frac{y}{\partial}}}{\partial \overline{\hat{y}}_{\bar{\alpha}_{h}}} \frac{\partial \overline{\hat{y}}_{\bar{\alpha}_{h}}}{\partial \bar{\theta}_{l}}+\frac{\partial \hat{y}}{\partial \overline{\hat{y}}} \frac{\partial \overline{\hat{y}}}{\partial \overline{\hat{y}}_{\underline{\alpha}_{h}}} \frac{\partial \overline{\hat{y}}_{\underline{\alpha}_{h}}}{\partial \bar{\theta}_{l}} \\
& =0.5 \frac{1}{\sum_{h=1}^{n}\left(\bar{\alpha}_{h}+\underline{\alpha}_{h}\right)^{h=1}} \sum^{n} \bar{\alpha}_{h} \frac{\bar{\varphi}_{\bar{\alpha}_{h}}^{l}}{\sum_{l=1}^{R}\left(\bar{\varphi}_{\underline{\alpha}_{h}}^{l}+\underline{\varphi}_{\alpha_{h}}^{l}\right)}+0.5 \frac{1}{\sum_{h=1}^{n}\left(\bar{\alpha}_{h}+\underline{\alpha}_{h}\right)} \sum_{h=1}^{n} \underline{\alpha}_{h} \frac{\bar{\varphi}_{\underline{\alpha}_{h}}^{l}}{\sum_{l=1}^{R}\left(\bar{\varphi}_{\underline{\alpha}_{h}}^{l}+\underline{\varphi}_{\underline{\alpha}_{h}}^{l}\right)} \\
& \underline{\psi}_{l}=\frac{\partial \hat{y}}{\partial \underline{\theta}_{l}}=\frac{\partial \hat{y}}{\partial \underline{\hat{y}}} \frac{\partial \underline{\hat{y}}}{\partial \underline{\theta}_{l}}=\frac{\partial \hat{y}}{\partial \underline{\hat{y}}} \frac{\partial \underline{\hat{y}}}{\partial \underline{\hat{y}}_{\bar{\alpha}_{h}}} \frac{\partial \underline{\hat{y}}_{\bar{\alpha}_{h}}}{\partial \underline{\theta}_{l}}+\frac{\partial \hat{y}}{\partial \underline{\hat{y}}} \frac{\partial \underline{\hat{y}}^{\hat{y}}}{\partial \underline{\hat{y}}_{\underline{\alpha}_{h}}} \frac{\partial \underline{\hat{y}}_{\underline{\alpha}_{h}}}{\partial \underline{\theta}_{l}} \\
& =0.5 \frac{1}{\sum_{h=1}^{n}\left(\bar{\alpha}_{h}+\underline{\alpha}_{h}\right)} \sum_{h=1}^{n} \bar{\alpha}_{h} \frac{\underline{\varphi}_{\bar{\alpha}_{h}}^{l}}{\sum_{l=1}^{R}\left(\bar{\varphi}_{\underline{\alpha}_{h}}^{l}+\underline{\varphi}_{\underline{\alpha}_{h}}^{l}\right)}+0.5 \frac{1}{\sum_{h=1}^{n}\left(\bar{\alpha}_{h}+\underline{\alpha}_{h}\right)} \sum_{h=1}^{n} \underline{\alpha}_{h} \frac{\underline{\varphi}_{\underline{\alpha}_{h}}^{l}}{\sum_{l=1}^{R}\left(\bar{\varphi}_{\underline{\alpha}_{h}}^{l}+\underline{\varphi}_{\underline{\alpha}_{h}}^{l}\right)}
\end{aligned}
$$




\subsection{Tuning of MF Parameters}

For the antecedent parameters, the centers of MFs are tuned on basis of gradient descent method. Then the tuning laws are written as:

$$
\begin{aligned}
& c_{\tilde{S}_{T}^{j}}(t)=c_{\tilde{S}_{T}^{j}}(t-1)-\gamma \frac{\partial J}{\partial c_{\tilde{S}_{T}^{j}}}, j=1,2 \\
& c_{\tilde{S}_{P}^{j}}(t)=c_{\tilde{S}_{P}^{j}}(t-1)-\gamma \frac{\partial J}{\partial c_{\tilde{S}_{P}^{j}}}, j=1,2 \\
& c_{\tilde{S}_{M}^{j}}(t)=c_{\tilde{S}_{M}^{j}}(t-1)-\gamma \frac{\partial J}{\partial c_{\tilde{S}_{M}^{j}}}, j=1,2
\end{aligned}
$$

where, $\gamma$ is the training rate. $\partial J / \partial c_{\tilde{S}_{T}^{1}}$ is obtained as follows:

$$
\begin{aligned}
& \frac{\partial J}{\partial c_{\bar{S}_{T}^{1}}}=\frac{\partial J}{\partial \hat{y}} \frac{\partial \hat{y}}{\partial \overline{\hat{y}}} \frac{\partial \overline{\hat{y}}}{\partial \overline{\hat{y}}_{\bar{\alpha}_{h}}}\left(\sum_{l=1}^{R} \bar{\zeta}_{T}^{l} \frac{\partial \overline{\hat{y}}_{\bar{\alpha}_{h}}}{\partial \bar{\varphi}_{\bar{\alpha}_{h}}^{l}} \frac{\partial \bar{\varphi}_{\bar{\alpha}_{h}}^{l}}{\partial c_{\bar{S}_{T}^{1}}}+\sum_{l=1}^{R} \bar{\zeta}_{T}^{l} \frac{\partial \overline{\hat{y}}_{\bar{\alpha}_{h}}}{\partial \underline{\varphi}_{\bar{\alpha}_{h}}^{l}} \frac{\partial \underline{\varphi}_{\bar{\alpha}_{h}}^{l}}{\partial c_{\bar{S}_{T}^{1}}}\right)+
\end{aligned}
$$

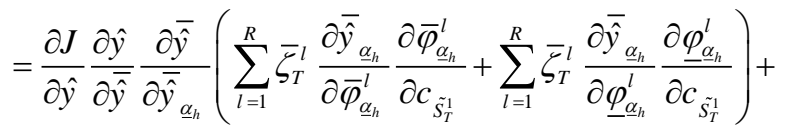

$$
\begin{aligned}
& =\frac{\partial J}{\partial \hat{y}} \frac{\partial \hat{y}}{\partial \underline{\hat{y}}} \frac{\partial \underline{\hat{y}}}{\partial \underline{\hat{y}}_{\underline{\alpha}_{h}}}\left(\sum_{l=1}^{R} \bar{\zeta}_{T}^{l} \frac{\partial \underline{\hat{y}}_{\underline{\alpha}_{h}}}{\partial \bar{\varphi}_{\underline{\alpha}_{h}}^{l}} \frac{\partial \bar{\varphi}_{\underline{\alpha}_{h}}^{l}}{\partial c_{\tilde{S}_{T}^{1}}}+\sum_{l=1}^{R} \bar{\zeta}_{T}^{l} \frac{\partial \underline{\hat{y}}_{\underline{\alpha}_{h}}}{\partial \underline{\varphi}_{\underline{\alpha}_{h}}^{l}} \frac{\partial \underline{\varphi}_{\underline{\alpha}_{h}}^{l}}{\partial c_{\tilde{S}_{T}^{1}}}\right)+ \\
& =\frac{\partial J}{\partial \hat{y}} \frac{\partial \hat{y}}{\partial \underline{\hat{y}}} \frac{\partial \underline{\hat{y}}}{\partial \underline{\hat{y}}_{\bar{\alpha}_{h}}}\left(\sum_{l=1}^{R} \bar{\zeta}_{T}^{l} \frac{\partial \underline{\hat{y}}_{\bar{\alpha}_{h}}}{\partial \bar{\varphi}_{\bar{\alpha}_{h}}^{l}} \frac{\partial \bar{\varphi}_{\bar{\alpha}_{h}}^{l}}{\partial c_{\bar{S}_{T}^{1}}}+\sum_{l=1}^{R} \bar{\zeta}_{T}^{l} \frac{\partial \underline{\hat{\alpha}}_{\bar{\alpha}_{h}}}{\partial \underline{\varphi}_{\bar{\alpha}_{h}}^{l}} \frac{\partial \underline{\varphi}_{\bar{\alpha}_{h}}^{l}}{\partial c_{\bar{S}_{T}^{1}}}\right)
\end{aligned}
$$

where, $\bar{\zeta}_{T}^{l}$ represents the $l-t h$ element of vector $\bar{\zeta}_{T}$. The vector $\bar{\zeta}_{T}$ is defined as:

$$
\bar{\zeta}_{T}=[1,1,1,1,0,0,0,0]
$$

where, the elements of $\bar{\zeta}_{T}$ in the rules that include $c_{\tilde{S}_{T}^{1}}$, are one. The terms $\frac{\partial \overline{\hat{y}}_{\bar{\alpha}_{h}}}{\partial \bar{\varphi}_{\bar{\alpha}_{h}}^{l}}$, $\frac{\partial \overline{\hat{y}}_{\bar{\alpha}_{h}}}{\partial \underline{\varphi}_{\bar{\alpha}_{h}}^{l}}, \frac{\partial \overline{\hat{y}}_{\underline{\alpha}_{h}}}{\partial \bar{\varphi}_{\underline{\alpha}_{h}}^{l}}, \frac{\partial \overline{\hat{y}}_{\underline{\alpha}_{h}}}{\partial \underline{\varphi}_{\underline{\alpha}_{h}}^{l}}, \frac{\partial \hat{\hat{y}}_{\bar{\alpha}_{h}}}{\partial \bar{\varphi}_{\bar{\alpha}_{h}}^{l}}, \frac{\partial \hat{\hat{\alpha}}_{\bar{\alpha}_{h}}}{\partial \underline{\varphi}_{\bar{\alpha}_{h}}^{l}}, \frac{\partial \hat{\hat{y}}_{\underline{\alpha}_{h}}}{\partial \bar{\varphi}_{\underline{\alpha}_{h}}^{l}}$ and $\frac{\partial \underline{\hat{y}}_{\underline{\alpha}_{h}}}{\partial \underline{\varphi}_{\underline{\alpha}_{h}}^{l}}$ are obtained as:

$$
\frac{\partial \overline{\hat{y}}_{\bar{\alpha}_{h}}}{\partial \bar{\varphi}_{\bar{\alpha}_{h}}^{l}}=\bar{\theta}_{l} \frac{\sum_{l=1}^{R}\left(\bar{\varphi}_{\bar{\alpha}_{h}}^{l}+\underline{\varphi}_{\bar{\alpha}_{h}}^{l}\right)-\bar{\varphi}_{\bar{\alpha}_{h}}^{l}}{\left(\sum_{l=1}^{R}\left(\bar{\varphi}_{\bar{\alpha}_{h}}^{l}+\underline{\varphi}_{\bar{\alpha}_{h}}^{l}\right)\right)^{2}}, \frac{\partial \overline{\hat{y}}_{\bar{\alpha}_{h}}}{\partial \underline{\varphi}_{\bar{\alpha}_{h}}^{l}}=\bar{\theta}_{l} \frac{-1}{\left(\sum_{l=1}^{R}\left(\bar{\varphi}_{\bar{\alpha}_{h}}^{l}+\underline{\varphi}_{\bar{\alpha}_{h}}^{l}\right)\right)^{2}}
$$




$$
\begin{aligned}
& \frac{\partial \overline{\hat{y}}_{\underline{\alpha}_{h}}}{\partial \bar{\varphi}_{{\underline{\alpha_{h}}}^{l}}^{l}}=\bar{\theta}_{l} \frac{\sum_{l=1}^{R}\left(\bar{\varphi}_{\underline{\alpha}_{h}}^{l}+\underline{\varphi}_{\underline{\alpha}_{h}}^{l}\right)-\bar{\varphi}_{\underline{\alpha}_{h}}^{l}}{\left(\sum_{l=1}^{R}\left(\bar{\varphi}_{\underline{\alpha}_{h}}^{l}+\underline{\varphi}_{\underline{\alpha}_{h}}^{l}\right)\right)^{2}}, \frac{\partial \underline{\hat{y}}_{\underline{\alpha}_{h}}}{\partial \underline{\varphi}_{\underline{\alpha}_{h}}^{l}}=\bar{\theta}_{l} \frac{-1}{\left(\sum_{l=1}^{R}\left(\bar{\varphi}_{\underline{\alpha}_{h}}^{l}+\underline{\varphi}_{\underline{\alpha}_{h}}^{l}\right)\right)^{2}} \\
& \frac{\partial \hat{\hat{y}}_{\bar{\alpha}_{h}}}{\partial \bar{\varphi}_{\bar{\alpha}_{h}}^{l}}=\underline{\theta}_{l} \frac{\sum_{l=1}^{R}\left(\bar{\varphi}_{\bar{\alpha}_{h}}^{l}+\underline{\varphi}_{\bar{\alpha}_{h}}^{l}\right)-\bar{\varphi}_{\bar{\alpha}_{h}}^{l}}{\left(\sum_{l=1}^{R}\left(\bar{\varphi}_{\bar{\alpha}_{h}}^{l}+\underline{\varphi}_{\bar{\alpha}_{h}}^{l}\right)\right)^{2}}, \frac{\partial \underline{\hat{y}}_{\bar{\alpha}_{h}}}{\partial \underline{\varphi}_{\bar{\alpha}_{h}}^{l}}=\underline{\theta}_{l} \frac{-1}{\left(\sum_{l=1}^{R}\left(\bar{\varphi}_{\bar{\alpha}_{h}}^{l}+\underline{\varphi}_{\bar{\alpha}_{h}}^{l}\right)\right)^{2}} \\
& \frac{\partial \underline{\hat{y}}_{\underline{\alpha}_{h}}}{\partial \bar{\varphi}_{\underline{\alpha}_{h}}^{l}}=\underline{\theta}_{l} \frac{\sum_{l=1}^{R}\left(\bar{\varphi}_{\underline{\alpha}_{h}}^{l}+\underline{\varphi}_{\underline{\alpha}_{h}}^{l}\right)-\bar{\varphi}_{\underline{\alpha}_{h}}^{l}}{\left(\sum_{l=1}^{R}\left(\bar{\varphi}_{\underline{\alpha}_{h}}^{l}+\underline{\varphi}_{\underline{\alpha}_{h}}^{l}\right)\right)^{2}}, \frac{\partial \underline{\hat{y}}_{\underline{\alpha}_{h}}}{\partial \underline{\varphi}_{\underline{\alpha}_{h}}^{l}}=\underline{\theta}_{l} \frac{-1}{\left(\sum_{l=1}^{R}\left(\bar{\varphi}_{\underline{\alpha}_{h}}^{l}+\underline{\varphi}_{\underline{\alpha}_{h}}^{l}\right)\right)^{2}}
\end{aligned}
$$

For $\frac{\partial \bar{\varphi}_{\bar{\alpha}_{h}}^{l}}{\partial c_{\tilde{S}_{T}^{1}}}, \frac{\partial \bar{\varphi}_{\underline{\alpha}_{h}}^{l}}{\partial c_{\tilde{S}_{T}^{1}}}, \frac{\partial \underline{\varphi}_{\bar{\alpha}_{h}}^{l}}{\partial c_{\tilde{S}_{T}^{1}}}$ and $\frac{\partial \underline{\varphi}_{\underline{\alpha}_{h}}^{l}}{\partial c_{\tilde{S}_{T}^{1}}}$ one has:

$$
\begin{aligned}
& \frac{\partial \bar{\varphi}_{\bar{\alpha}_{h}}^{l}}{\partial c_{\tilde{S}_{T}^{1}}}=\frac{2\left(T-c_{\bar{S}_{T}^{1} \mid \bar{\alpha}_{h}}\right)}{\bar{\sigma}_{\tilde{S}_{T}^{1} \mid \bar{\alpha}_{h}}^{2}} \bar{\varphi}_{\bar{\alpha}_{h}}^{l}, \frac{\partial \bar{\varphi}_{\underline{\alpha}_{h}}^{l}}{\partial c_{\tilde{S}_{T}^{1}}}=\frac{2\left(T-c_{\tilde{S}_{T}^{1} \mid \underline{\alpha}_{h}}\right)}{\bar{\sigma}_{\tilde{S}_{T}^{1} \mid \underline{\alpha}_{h}}^{2}} \bar{\varphi}_{\underline{\alpha}_{h}}^{l} \\
& \frac{\partial \underline{\varphi}_{\bar{\alpha}_{h}}^{l}}{\partial c_{\tilde{S}_{T}^{1}}}=\frac{2\left(T-c_{\tilde{S}_{T}^{1} \mid \bar{\alpha}_{h}}\right)}{\bar{\sigma}_{\tilde{S}_{T}^{1} \mid \bar{\alpha}_{h}}^{2}} \underline{\varphi}_{\bar{\alpha}_{h}}^{l}, \frac{\partial \underline{\varphi}_{\alpha_{h}}^{l}}{\partial c_{\tilde{S}_{T}^{1}}}=\frac{2\left(T-c_{\tilde{S}_{T}^{1} \mid \underline{\alpha}_{h}}\right)}{\bar{\sigma}_{\tilde{S}_{T}^{1} \mid \underline{\alpha}_{h}}^{2}} \underline{\varphi}_{\underline{\alpha}_{h}}^{l}
\end{aligned}
$$

The computation for terms $\partial J / \partial c_{\tilde{S}_{T}^{2}}, \partial J / \partial c_{\tilde{S}_{M}^{1}}, \partial J / \partial c_{\tilde{S}_{M}^{2}}, \partial J / \partial c_{\tilde{S}_{P}^{1}}$ and $\partial J / \partial c_{\tilde{S}_{P}^{2}}$, are the same as $\partial J / \partial c_{\tilde{S}_{T}^{1}}$, with difference that $\bar{\zeta}_{T}$ is repealed with $\underline{\zeta}_{T}$, $\bar{\zeta}_{M}, \underline{\zeta}_{M}, \bar{\zeta}_{P}$ and $\underline{\zeta}_{P}$, respectively. Also terms $c_{\tilde{S}_{T}^{1} \mid \bar{\alpha}_{h}}, c_{\tilde{S}_{T}^{1} \mid \underline{\alpha}_{h}}, \bar{\sigma}_{\tilde{S}_{T}^{1} \mid \bar{\alpha}_{h}}$ and $\bar{\sigma}_{\tilde{S}_{T}^{1} \mid \underline{\alpha}_{h}}^{2}$ should be replaced with the corresponding terms. The vectors $\underline{\zeta}_{T}, \bar{\zeta}_{M}, \underline{\zeta}_{M}, \bar{\zeta}_{P}$ and $\underline{\zeta}_{P}$ are defined as:

$$
\begin{aligned}
& \underline{\zeta}_{T}=[0,0,0,0,1,1,1,1], \bar{\zeta}_{M}=[1,0,1,0,1,0,1,0] \\
& \underline{\zeta}_{M}=[0,1,0,1,0,1,0,1], \bar{\zeta}_{P}=[1,1,0,0,1,1,0,0], \underline{\zeta}_{P}=[0,0,1,1,0,0,1,1]
\end{aligned}
$$




\subsection{Optimizing of Horizontal Slices}

For the optimizing of horizontal slices level, the UKF algorithm is used. To apply UKF algorithm the state space of the suggested T3-FLS is written as follows:

$\alpha(t+1)=\alpha(t)+v(t)$

$\hat{y}(t+1)=\mathrm{T} 3-\mathrm{FLS}(u(t)|\theta(t)| \alpha(t))+v(t)$

where, $v(t)$ and $v(t)$ represent noise with covariance $v_{p}$ and $v_{m}$, respectively. $u(t)$, $\theta(t)$ and $\alpha(t)$ are the vectors of input variables, consequent parameters and values of horizontal slices, respectively. The sigma points $\tilde{\alpha}$ are computed as:

$\tilde{\alpha}_{z}=\tilde{\alpha}_{z}+\tilde{w}_{z}, z=1, \ldots, 2 n$

where, $n$ is the number of $\alpha$-cuts and

$\tilde{w}_{z}=(\sqrt{n \psi(t)})^{T}, z=1, \ldots, n$
$\tilde{w}_{z+n}=-(\sqrt{n \psi(t)})^{T}, z=1, \ldots, n$

where, $\psi(t)$ is the covariance matrix. For each $\tilde{\alpha}(t)$ in (46), the output of T3FLS are computed as:

$\tilde{y}_{z}(t+1)=\mathrm{T} 3-\mathrm{FLS}\left(u(t)|\theta(t)| \tilde{\alpha}_{z}(t)\right)$

From (49), the average $\bar{y}$ is:

$$
\bar{y}=\sum_{z=1}^{2 n} \tilde{y}_{z} /(2 n)
$$

The cross-covariance $\psi_{\alpha y}$ is obtained as:

$\psi_{\alpha y}=\frac{1}{2 n} \sum_{z=1}^{2 n}\left(\tilde{\alpha}_{z}-\hat{\alpha}\right) \bar{y}$

where, $\hat{\alpha}$ is computed as:

$\hat{\alpha}=\frac{1}{2 n} \sum_{z=1}^{2 n} \tilde{\alpha}_{z}$

Kalman gain is computed as:

$K(t)=\psi_{\alpha y} \psi^{-1}$

Finally, the vector of $\alpha$-cuts are updated as:

$\alpha(t+1)=\alpha(t)-K(t) \bar{y}(t)$ 


\section{Evaluation Indexes and Data Description}

To examine the performance of the suggested method, 550 real-world data are collected from [7, 38]. The maximum of pressure, molality, temperature, and solubility are $1400,6.14,723.15$, and 12.35 , respectively. The minimum of pressure, molality, temperature, and solubility are $0.98,0.016,273.15$, and 0.01 , respectively. The data is normalized into the range $[0,1] .80 \%$ of data is randomly selected for training process and remains for testing.

To examine the capability of the suggested method, the following indexes are employed:

$$
\begin{aligned}
& \mathrm{RMSE}=\sqrt{\frac{1}{N} \sum_{i=1}^{N}\left(y_{i}-\hat{y}_{i}\right)^{2}}, \mathrm{VAR}=\sum_{i=1}^{N}\left(y-\hat{y}_{i}\right)^{2} /(N-1) \\
& \mathrm{TIC}=\frac{\sqrt{\frac{1}{N} \sum_{i=1}^{N}\left(y_{i}-\hat{y}_{i}\right)^{2}}}{\sqrt{\frac{1}{N} \sum_{i=1}^{N} y_{i}^{2}}+\sqrt{\frac{1}{N} \sum_{i=1}^{N} \hat{y}_{i}^{2}}}, \mathrm{R}^{2}=1-\frac{\sum_{i=1}^{N}\left(y_{i}-\hat{y}_{i}\right)^{2}}{\sum_{i=1}^{N}\left(\frac{1}{N} \sum_{i=1}^{N} y_{i}-\hat{y}_{i}\right)^{2}}
\end{aligned}
$$

where, $N$ represents the number of data, $y_{i}$ is the real solubility, $\hat{y}_{i}$ is the estimated solubility and RMSE, TIC and VAF are root mean square error, Theil's inequality coefficient, and variance account for, respectively.

\section{Simulation}

In this section, the performance of the suggested T3-FLS and hybrid learning algorithm is examined.

\subsection{Results for Testing Data}

To examine the estimation performance of the suggested T3-FLS and learning algorithm several statistical analyses were presented. Estimation performance for test and training data are shown in Figure 4. It is seen that the estimated solubility is well converged to the real one and the estimation error is at a desired and logical level. 


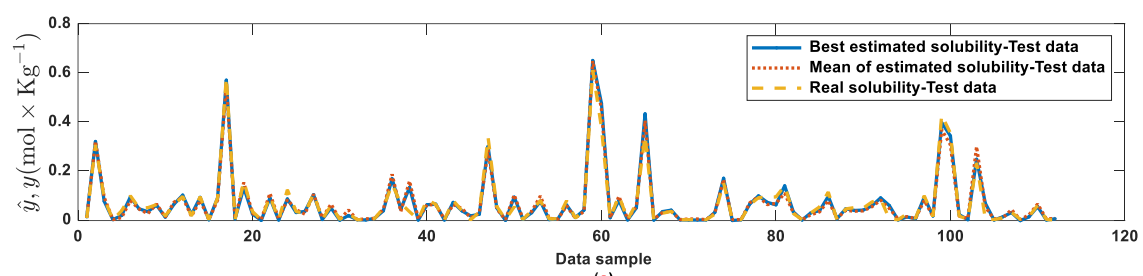

(a)

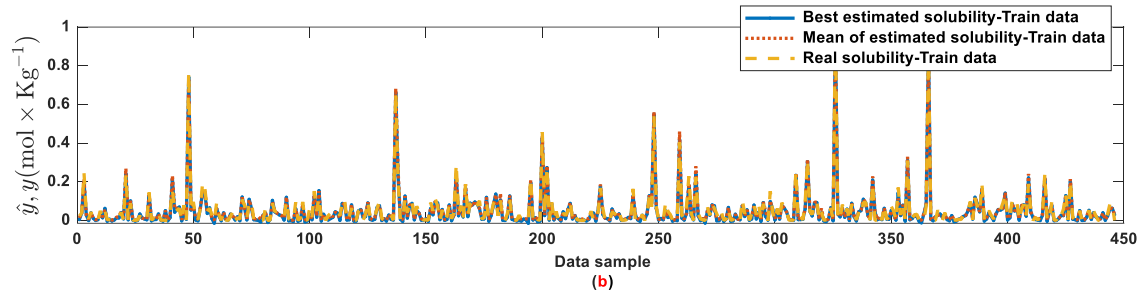

Figure 4

(a): Estimation performance for testing data ; (b): Estimation performance for training data

The absolute error for testing data and the values of RMSE, VAR, and TIC for testing data are given in Figure 5. One can see that the maximum and of the absolute error in the worst state is less than 2 .
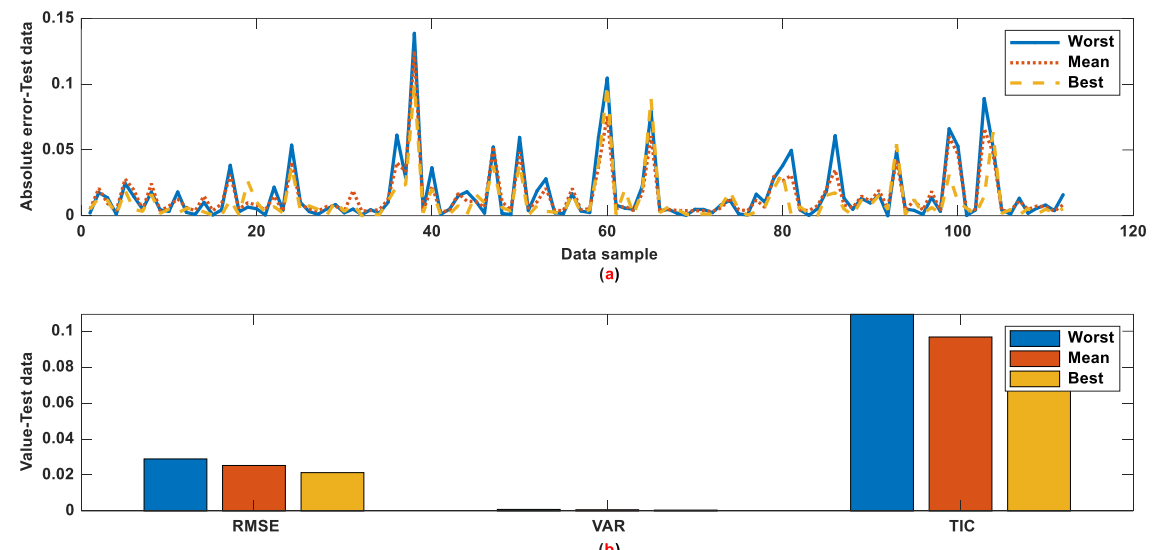

Figure 5

(a): Absolute error for testing data; (b): The values of RMSE, VAR and TIC for testing data 

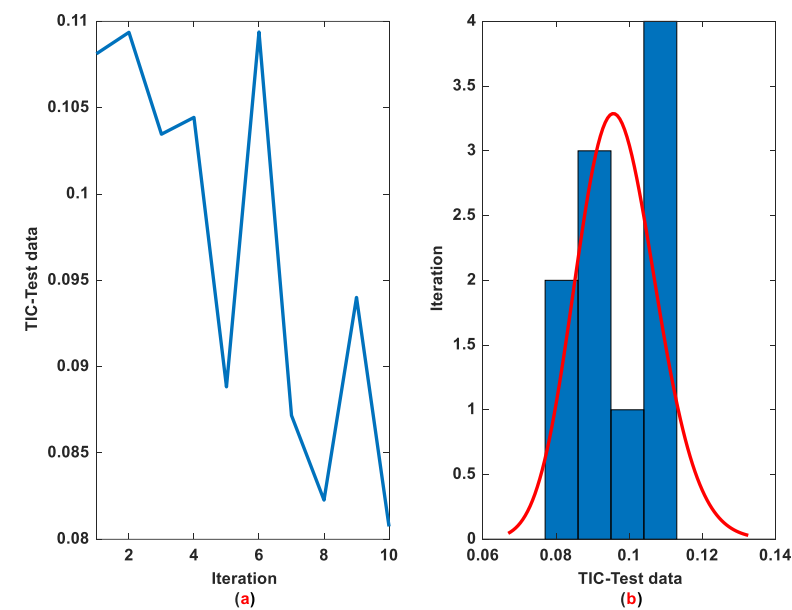

Figure 6

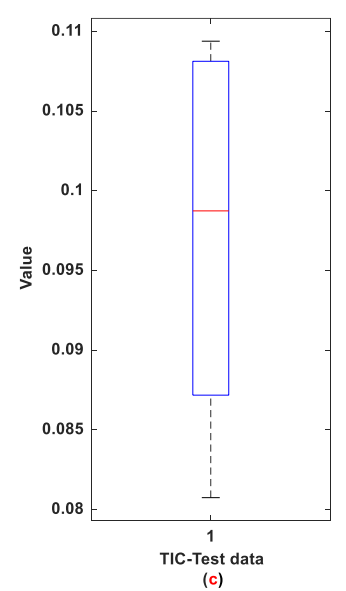

(c)

(a): The value of TIC for testing data; (b): Histogram diagram for TIC for testing data; (c): Box plot of TIC for testing data

The value of TIC, the histogram plot for TIC, and Box plot of TIC for testing data are depicted in Figure 6. It is seen that the mean of TIC is about 0.08, and the maximum of TIC is less than 0.09. The value of RMSE at each iteration, the histogram plot of RMSE, and Box plot of RMSE for testing data are shown in Figure 5. The mean of RMSE is about 0.25 and the maximum of RMSE is less than 0.26 . The value of VAR at each iteration, the histogram plot of VAR, and the Box plot of VAR for testing data are given in Figure 8. It is seen that the value of VAR is small enough and the results in various iterations are close to each other. The values of R2 in iterations are shown in Figure 9. As it is seen the average of $\mathrm{R} 2$ is greater than 0.95 , which represents a good correlation. 


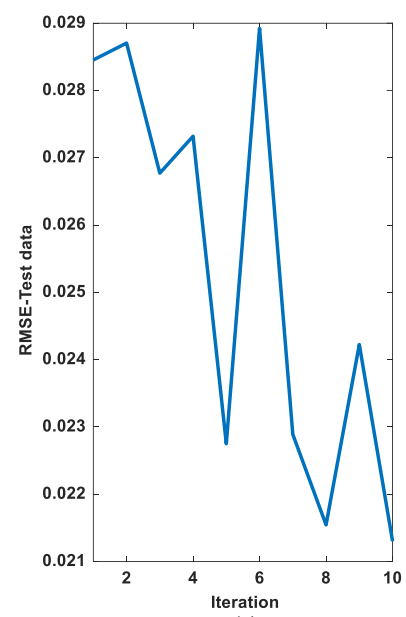

(a)

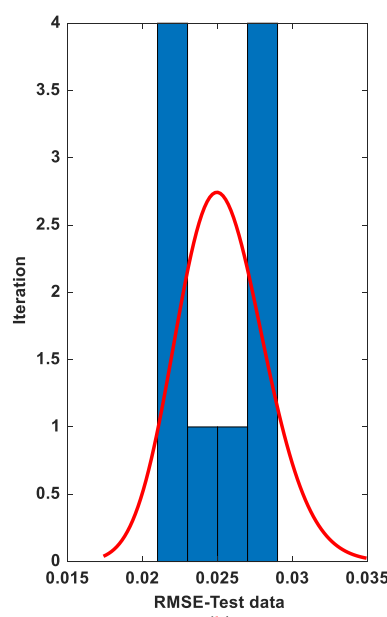

(b)

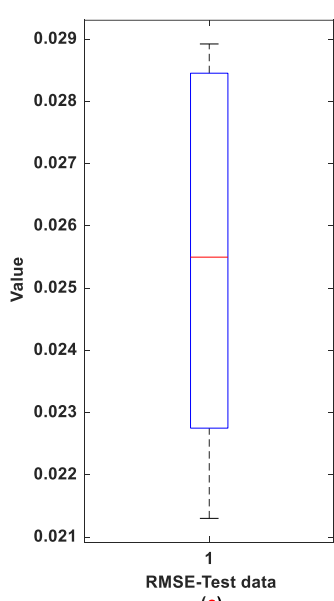

(c)

Figure 7

(a): The value of RMSE at each iteration for testing data; (b): Histogram plot of RMSE for testing data; (c): Box plot of RMSE for testing data

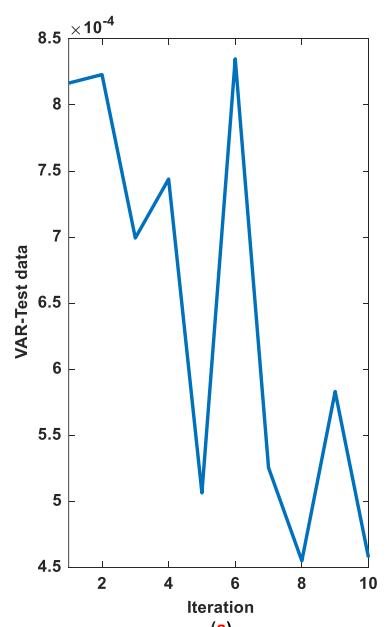

(a)

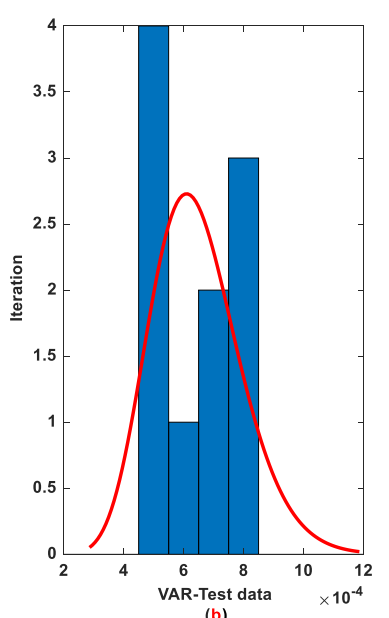

(b)

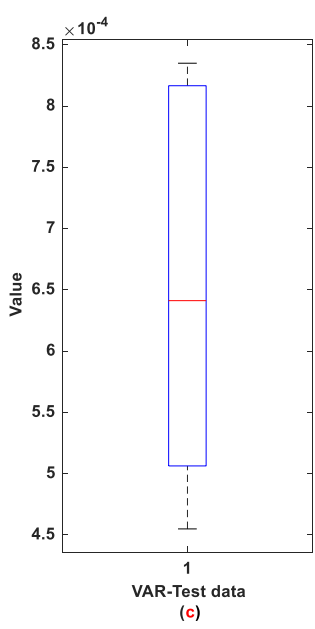

(c)

Figure 8

(a): The value of VAR at each iteration for testing data; (b): Histogram plot of VAR for testing data;

(c): Box plot of VAR for testing data 


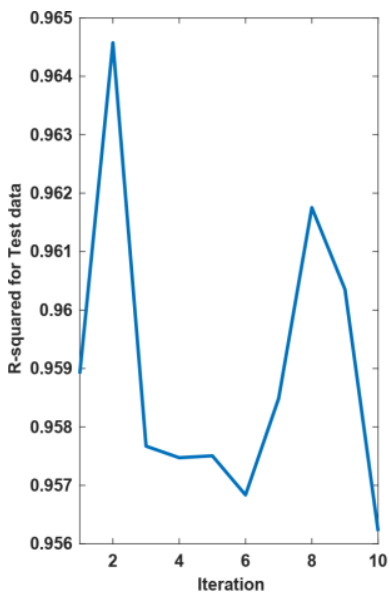

(a)

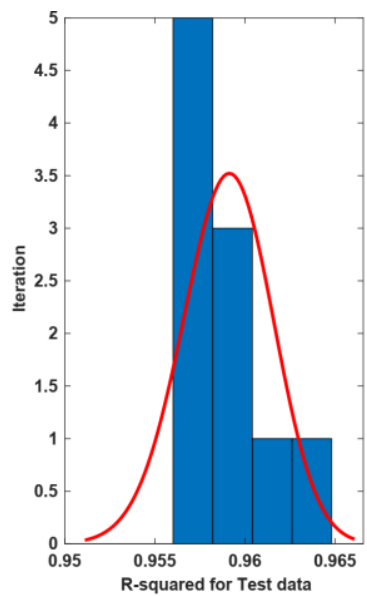

(b)

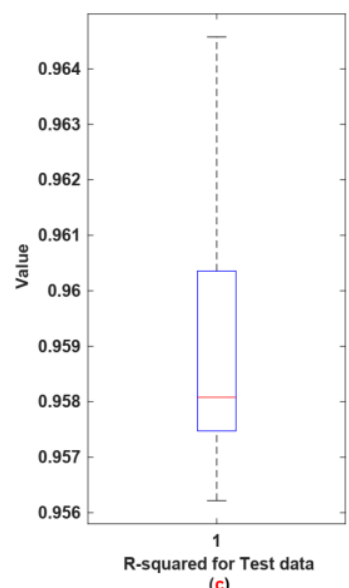

(c)

Figure 9

(a): The value of R2 at each iteration for testing data; (b): Histogram plot of R2 for testing data; (c):

Box plot of R2 for testing data

\subsection{Sensitivity Analysis}

For sensitivity analysis, the Sobol method is employed. In this approach, the variance of the solubility is decomposed on its input variance. The sensitivity index is a value between 0 and 1 . The larger index represents the higher influence. The values of the first-order sensitivity index for the suggested T3-FLS model are given in Table 1. As it is seen the most effective variable of solubility is the pressure.

Table 1

Sensitivity analysis by Sobol approach

\begin{tabular}{|c|c|c|c|}
\hline Structure & Temperature & Pressure & Molality of N \\
\hline First-Order Sensitivity Effect & 0.0396 & 0.2291 & 0.1256 \\
\hline
\end{tabular}

\subsection{Comparison and Discussion}

A comparison of RMSE with various neuro-fuzzy systems is given in Table 2. The performance of the suggested method is compared with the MLP, RBF, type1 FLS (T1-FLS), and type-2 FLS (T2-FLS). It is observed that the suggested T3FLS results in better solubility perdition performance. To better show the accommodation between model output and measured data, the cross plot for train and testing data is depicted in Figure 10. It is seen that most of the training and testing data are near the unit slope line. This plot verifies the good estimation 
performance and well accommodation. Furthermore, a comparison with other learning methods is given in Table 3 . The proposed hybrid learning system is compared with GA, PSO, and ABC algorithms that are frequently used in literature for optimization of models of $\mathrm{CO} 2$ solubility. It is seen that the suggested hybrid learning method results in better accuracy.

Table 2

Comparison of RMSE with various neuro-fuzzy systems

\begin{tabular}{|c|c|c|c|c|c|}
\hline Structure & MLP & RBF & T1-FLS & T2-FLS & T3-FLS \\
\hline RMSE & 0.34 & 0.33 & 0.31 & 0.27 & 0.23 \\
\hline $\mathrm{R}^{2}$ & 0.81 & 0.82 & 0.83 & 0.85 & 0.95 \\
\hline
\end{tabular}

Table 3

Comparison of RMSE with various learning algorithms

\begin{tabular}{|c|c|c|c|c|}
\hline $\begin{array}{c}\text { Learning } \\
\text { Method }\end{array}$ & PSO & GA & ABC & Proposed \\
\hline RMSE & 0.31 & 0.30 & 0.28 & 0.23 \\
\hline
\end{tabular}

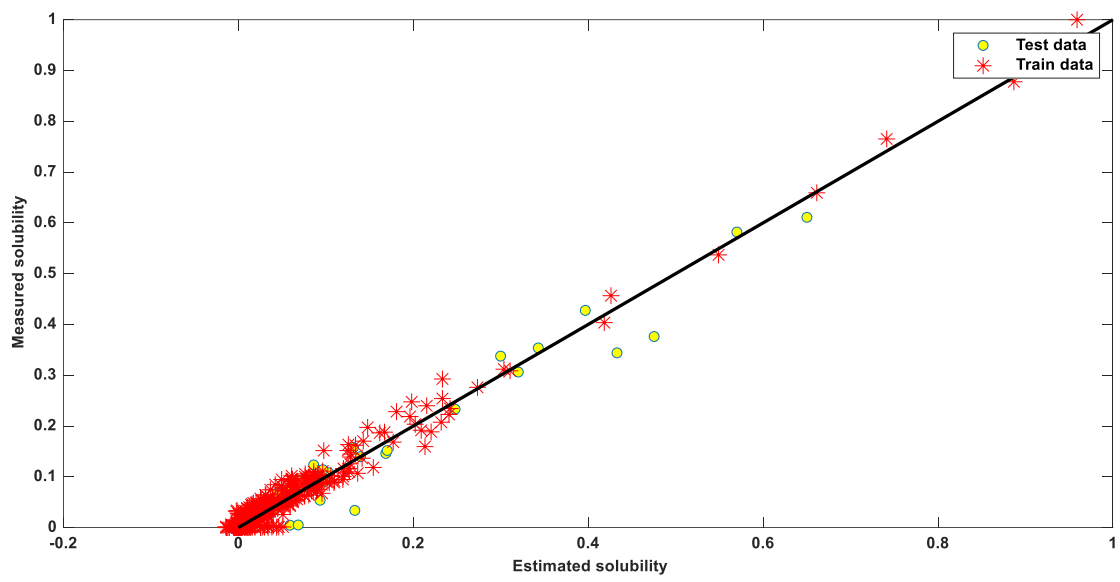

Figure 10

Cross plot for train and testing data

\section{Conclusions}

In this study, a new approach on basis of type-3 FLSs is proposed to construct a model between solubility and affective variables such as temperature, pressure, and molality of $\mathrm{NaCl}$. The parameters of the suggested model are tuned by a hybrid learning method using EKF and UKF. The rule and centers of membership functions are optimized by EKF and the level of horizontal slices are tuned by the UKF algorithm. Several statistical analyses such as plotting the trajectories of TIC, VAR, RMSE, Box plot, R-squared, and histogram plot are provided to 
demonstrate the effectiveness and reliability of the suggested method. It is shown that the estimation performance in various initial conditions does not change significantly. Also, two comparisons with conventional well-known structures and well-known learning algorithms demonstrate the superiority of the presented T3FLS and learning algorithm. Furthermore, sensitivity analysis by the Sobol approach is provided to show the most effective input variable of T3-FLS.

\section{References}

[1] M. M. Balas, Seven Passive Greenhouse Synergies, Acta Polytechnica Hungarica 11 (4) (2014) 199-210

[2] E. Ali, M. K. Hadj-Kali, S. Mulyono, I. Alnashef, A. Fakeeha, F. Mjalli, A. Hayyan, Solubility of $\mathrm{co} 2$ in deep eutectic solvents: experiments and modelling using the peng-robinson equation of state, Chemical Engineering Research and Design 92 (10) (2014) 1898-1906, https://doi.org/10.1016/j.cherd.2014.02.004

[3] N. I. Diamantonis, G. C. Boulougouris, D. M. Tsangaris, M. J. El Kadi, H. Saadawi, S. Negahban, I. G. Economou, Thermodynamic and transport property models for carbon capture and sequestration (ccs) processes with emphasis on co2 transport, Chemical engineering research and design 91 (10) (2013) 1793-1806, https://doi.org/10.1016/j.cherd.2013.06.017

[4] D. Allen, B. Strazisar, Y. Soong, S. Hedges, Modeling carbon dioxide sequestration in saline aquifers: Significance of elevated pressures and salinities, Fuel processing technology 86 (14-15) (2005) 1569-1580, https://doi.org/10.1016/j.fuproc.2005.01.004

[5] A. Riaz, Y. Cinar, Carbon dioxide sequestration in saline formations: Part I review of the modeling of solubility trapping, Journal of Petroleum Science and Engineering $124 \quad$ (2014) 367-380, https://doi. org/10.1016/j.petrol.2014.07.024

[6] A. Hassanpouryouzband, M. V. Farahani, J. Yang, B. Tohidi, E. Chuvilin, V. Istomin, B. Bukhanov, Solubility of flue gas or carbon dioxide-nitrogen gas mixtures in water and aqueous solutions of salts: Experimental measurement and thermodynamic modeling, Industrial \& Engineering $\begin{array}{lllll}\text { Chemistry } & \text { Research } & 58 & \text { (8) } & \text { (2019) 3377-3394, }\end{array}$ https://doi.org/10.1021/acs.iecr.8b04352

[7] W. Yan, S. Huang, E. H. Stenby, Measurement and modeling of co2 solubility in nacl brine and co2- saturated nacl brine density, International Journal of Greenhouse Gas Control 5 (6) (2011) 1460-1477, https://doi.org/10.1016/j.ijggc.2011.08.004

[8] H. Zhao, R. Dilmore, D. E. Allen, S. W. Hedges, Y. Soong, S. N. Lvov, Measurement and modeling of co2 solubility in natural and synthetic formation brines for co2 sequestration, Environmental science \& technology 49 (3) (2015) 1972-1980, https://doi.org/10.1021/es505550a 
[9] A. Chapoy, A. Mohammadi, A. Chareton, B. Tohidi, D. Richon, Measurement and modeling of gas solubility and literature review of the properties for the carbon dioxide- water system, Industrial \& engineering

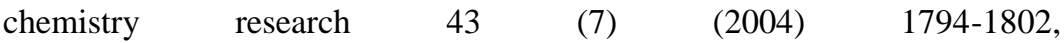
https://doi.org/10.1021/ie034232t

[10] S. Chabab, P. Theveneau,' J. Corvisier, C. Coquelet, P. Paricaud, C. ouriez, E. El Ahmar, Thermodynamic study of the co2-h2o-nacl system: easurements of co2 solubility and modeling of phase equilibria using soreide and whitson, electrolyte cpa and sit models, International Journal of $\begin{array}{lllll}\text { reenhouse Gas } & \text { Control } & 91 & \text { (2019) }\end{array}$ https://doi.org/10.1016/j.ijggc.2019.102825

[11] G. Sodeifian, N. S. Ardestani, S. A. Sajadian, Solubility measurement of a pigment (phthalocyanine green) in supercritical carbon dioxide: Experimental correlations and thermodynamic modeling, Fluid Phase Equilibria 494 (2019) 61-73, https://doi.org/10.1016/j.fluid.2019.04.024

[12] A. Pejic,' P. S. Molcer, Predictive machine learning approach for complex problem solving process data mining, Acta Polytechnica Hungarica 18 (1)

[13] A. Di Noia, A. Martino, P. Montanari, A. Rizzi, Supervised machine learning techniques and genetic optimization for occupational diseases risk prediction, Soft Computing 24 (6) (2020) 4393-4406

[14] G. Grmanova,' P. Laurinec, V. Rozinajova,' A. B. Ezzeddine, M. Lucka,' P. Lacko, P. Vrablecova,' P. Navrat,' Incremental ensemble learning for electricity load forecasting, Acta Polytechnica Hungarica 13 (2) (2016) 97117

[15] T. T. Ngoc, C. M. T. Le Van Dai, C. M. Thuyen, Support vector regression based on grid search method of hyperparameters for load forecasting, Acta Polytechnica Hungarica 18 (2) (2021) 143-158

[16] H. Moayedi, A. Osouli, D. T. Bui, L. Kok Foong, H. Nguyen, B. Kalantar, Two novel neural-evolutionary predictive techniques of dragonfly algorithm (da) and biogeography-based optimization (bbo) for landslide susceptibility analysis, Geomatics, Natural Hazards and Risk 10 (1) (2019) 2429-2453

[17] T. Stepisnik, D. Kocev, S. Dzeroski, ${ }^{\smile}$ Option predictive clustering trees for multi-label classification, Acta Polytechnica Hungarica 17 (10)

[18] A. Khamis, J. Meng, J. Wang, A. T. Azar, E. Prestes, H. Li, I. A. Hameed, A. Takacs,' I. J. Rudas, T. Haidegger, Robotics and intelligent systems against a pandemic, Acta Polytechnica Hungarica 18 (5)

[19] M. Raji, A. Dashti, P. Amani, A. H. Mohammadi, Efficient estimation of co2 solubility in aqueous salt solutions, Journal of Molecular Liquids 283 (2019) 804-815, https://doi.org/10.1016/j.molliq.2019.02.090 
[20] A. Baghban, A. Bahadori, A. H. Mohammadi, A. Behbahaninia, Prediction of $\operatorname{co} 2$ loading capacities of aqueous solutions of absorbents using different computational schemes, International Journal of Greenhouse Gas Control 57 (2017) 143-161, https://doi.org/10.1016/j.ijggc.2016.12.010

[21] H. Saghafi, M. Arabloo, Modeling of co2 solubility in mea, dea, tea, and mdea aqueous solutions using adaboost-decision tree and artificial neural network, International Journal of Greenhouse Gas Control 58 (2017) 256265, https://doi.org/10.1016/j.ijggc.2016.12.014

[22] H. Saghafi, M. M. Ghiasi, A. H. Mohammadi, Co2 capture with aqueous solution of sodium glycinate: Modeling using an ensemble method, International Journal of Greenhouse Gas Control 62 (2017) 23-30, https://doi.org/10.1016/j.ijggc.2017.03.029

[23] H. Yarveicy, H. Saghafi, M. M. Ghiasi, A. H. Mohammadi, Decision treebased modeling of co2 equilibrium absorption in different aqueous solutions of absorbents, Environmental Progress \& Sustainable Energy 38 (s1) (2019) S441-S448, https://doi.org/10.1002/ep.13128

[24] S.-A. Hoseinpour, A. Barati-Harooni, P. Nadali, A. Mohebbi, A. NajafiMarghmaleki, A. Tatar, A. Bahadori, Accurate model based on artificial intelligence for prediction of carbon dioxide solubility in aqueous tetra-nbutylammonium bromide solutions, Journal of Chemometrics 32 (2) (2018) e2956, https://doi.org/10.1002/cem.2956

[25] A. Baghban, H. Rajabi, N. Jamshidi, ANFIS modeling of carbon dioxide capture from gas stream emissions in the petrochemical production units, Petroleum Science and Technology 35 (6) (2017) 625-631, https://doi.org/10.1080/10916466.2016.1273241

[26] A. Mardani, H. Liao, M. Nilashi, M. Alrasheedi, F. Cavallaro, A multistage method to predict carbon dioxide emissions using dimensionality reduction, clustering, and machine learning techniques, Journal of Cleaner Production $275 \quad$ (2020) 122942, https://doi.org/10.1016/j.jclepro.2020.122942

[27] A. Daryasafar, A. Keykhosravi, K. Shahbazi, Modeling co2 wettability behavior at the interface of brine/co2/mineral: Application to co2 geosequestration, Journal of Cleaner Production 239 (2019) 118101, https://doi.org/10.1016/j.jclepro.2019.118101

[28] Y. Wang, Toward modeling of solubility of carbon dioxide, methane, and nitrogen in liquid dibenzyl toluene (ldt) using rigorous technique, Energy Sources, Part A: Recovery, Utilization, and Environmental Effects (2019) 1-10, https://doi.org/10.1080/15567036.2019.1694100

[29] M. N. Amar, M. A. Ghriga, H. Ouaer, M. E. A. B. Seghier, B. T. Pham, P. $\varnothing$. Andersen, Modeling viscosity of $\mathrm{co} 2$ at high temperature and pressure 
conditions, Journal of Natural Gas Science and Engineering 77 (2020) 103271, https://doi.org/10.1016/j.jngse.2020.103271

[30] A. Bemani, A. Baghban, A. Mosavi, Estimating co2-brine diffusivity using hybrid models of anfis and evolutionary algorithms, Engineering Applications of Computational Fluid Mechanics 14 (1) (2020) 818-834, https://doi.org/10.1080/19942060.2020.1774422

[31] M. Afkhami Karaei, B. Honarvar, A. Azdarpour, E. Mohammadian, On prediction of carbon dioxide solubility in aqueous systems of nacl using lssvm algorithm, Energy Sources, Part A: Recovery, Utilization, and Environmental Effects (2019) 1-10, https://doi.org/10.1080/15567036.2019.1651789

[32] Q. Feng, R. Cui, S. Wang, J. Zhang, Z. Jiang, Estimation of co2 diffusivity in brine by use of the genetic algorithm and mixed kernels-based support vector machine model, Journal of Energy Resources Technology 141 (4), https://doi.org/10.1115/1.4041724

[33] Wang H, Liu DH, Yu L, Chen GJ, Yan HP, Tian MW, Huang HL, Yan SR, Wang LN, Li WH. Analysis on the reading guidance to classics in the teaching of specialized courses in tourism undergraduate education in China. The International Journal of Electrical Engineering \& Education. 2020 Jun 30:0020720920931073

[34] Tian M, Yan S, Tian X. Discrete approximate iterative method for fuzzy investment portfolio based on transaction cost threshold constraint. Open Physics. 2019 Jan 1;17(1):41-7

[35] Tian MW, Wang S, Yan SR. Damage Assessment of Marine Pollution Disasters to Biological Resources Based on Numerical Models. Acta Microscopica. 2019 Apr 1;28(3)

[36] Tian MW, Yan SR, Tian XX, Liu JA. Research on image recognition method of bank financing bill based on binary tree decision. Journal of Visual Communication and Image Representation. 2019 Apr 1;60:123-8

[37] A. Mohammadzadeh, M. H. Sabzalian, W. Zhang, An interval type-3 fuzzy system and a new online fractional-order learning algorithm: theory and practice, IEEE Transactions on Fuzzy Systems 28 (2019) 1940-1950, https://doi.org/10.1109/TFUZZ.2019.2928509

[38] M. S. Aktar, M. De, S. Maity, S. K. Mazumder, M. Maiti, Green 4d transportation problems with breakable incompatible items under type-2 fuzzy-random environment, Journal of Cleaner Production 275 (2020) 122376 